Editor's Note: These short reviews of a recent paper in the Journal, written exclusively by graduate students or postdoctoral fellows, are intended to mimic the journal clubs that exist in your own departments or institutions. For more information on the format and purpose of the Journal Club, please see http://www.jneurosci.org/misc/ifa_features.shtml.

\title{
TRPA1: The Central Molecule for Chemical Sensing in Pain Pathway?
}

\author{
Chao Tai, Shanshan Zhu, and Ning Zhou \\ Brain Research Center, University of British Columbia, Vancouver, British Columbia, Canada V6T2B5 \\ Review of Macpherson et al. (http://www.jneurosci.org/cgi/content/full/27/42/11412)
}

In mammals, potentially dangerous signals from the environment are detected by neurons in the pain pathway. Specialized neurons within the peripheral nervous system called nociceptive neurons sense noxious chemical, thermal, or mechanical stimuli and transmit signals to the CNS. It is well known that injurious stimuli such as tissue damage and inflammation trigger the generation of numerous molecules, including peptides (bradykinin), lipids (prostaglandins), and many other small molecules in the affected regions. These compounds could significantly depolarize the nerve terminals of nociceptors, which send danger signals to the brain (Fig. 1A) (Julius and Basbaum, 2001). Despite extensive studies, however, it is still not clear which channels convert chemical signals to conductances. To date, several candidate ion channels have been suggested, including ATP-gated channels (P2X receptors), acid-gated channels (ASICs), and vanilloid transient receptor potential (TRP) channels (Julius and Basbaum, 2001), as shown in Figure $1 B$. Nonetheless, it is still a mystery why, although numerous chemicals can induce pain, none of the channels described to

\footnotetext{
Received Nov. 26, 2007; revised Dec. 15, 2007; accepted Dec. 27, 2007.

We thank Dr. Brian A. MacVicar and Dr. Hyun Beom Choi for helpful comments on this manuscript.

Correspondence should be addressed to Chao Tai, Brain Research Center, 2211 Wesbrook Mall, Vancouver, British Columbia, Canada V6T2B5. E-mail: tai_chao@hotmail.com.

DOI:10.1523/JNEUROSCI.5237-07.2008

Copyright $\odot$ 2008 Society for Neuroscience $\quad$ 0270-6474/08/281019-03\$15.00/0
}

date can sense diverse compounds and integrate information from diverse chemicals. Excitingly, in a recent paper published in the Journal of Neuroscience, Macpherson et al. (2007b) provide convincing data showing that a member of the TRP family, TRPA1 (ANKTM1), displays broad specificity to many chemically damaging molecules both in vitro and in vivo, and functions as a central chemical nocisensor.

TRP channels form a recently identified superfamily of cation channels that display great diversity of activation mechanisms and selectivities (Venkatachalam and Montell, 2007). They are widely distributed in mammalian tissues, and they respond to a wide range of environmental signals, including temperature, touch, pain, osmolarity, taste, $\mathrm{pH}$, pheromones, and other stimuli (Venkatachalam and Montell, 2007). Despite extensive studies on these channels, two central questions are still largely unanswered: (1) How are these channels gated? (2) What are their specific physiological roles? Encouragingly, Macpherson et al. (2007b) provide convincing evidence to answer both of the questions with respect to at least one group of TRPs, the TRPA1 channels.

TRPA1 is the sole member of a distinct TRP family that was initially reported to sense noxious cold (Story et al., 2003) (but see also Jordt et al., 2004). TRPA1 channels can be chemically activated by many exogenous small organic molecules, including ingredients in wasabi, horserad- ish, marijuana, garlic, and mustard oil (Venkatachalam and Montell, 2007). However, to date, no endogenous chemical activators from the aqueous extracellular milieu have been identified. In their Figure 1 (http://www.jneurosci.org/cgi/ content/full/27/42/11412/F1), the authors tested many chemicals for their ability to activate TRPA1 channels, using ratiometric $\mathrm{Ca}^{2+}$ imaging and fluorometric imaging plate reader techniques. They found that formaldehyde, a common compound to induce chemical pain, could activate TRPA1 channels. More interestingly, they found that an endogenous compound, 4-hydroxynonenal (4-HNE; an $\alpha, \beta$-unsaturated aldehyde that accumulates in membranes during inflammatory or oxidative stress), could also activate TRPA1 channels, at physiological concentration $\left(\mathrm{EC}_{50}=13 \mu \mathrm{M}\right)$. Using inside-out single-channel patchclamp technique, they confirmed the activation ability of these compounds on TRPA1 currents [Macpherson et al. (2007b), their Fig. 2 (http://www. jneurosci.org/cgi/content/full/27/42/ $11412 / \mathrm{F} 2)]$. This is the first time that endogenous molecules produced during tissue damage or oxidative stress (i.e., 4-HNE and its analogs) have been shown to activate TRP channels [it should be noted that recently another group independently reported nearly identical results about the action of 4-HNE on TRPA1 (Trevisani et al., 2007)], suggesting the existence of the 
endogenous ligands for TRPA1 and other TRP channels.

Next, Macpherson et al. (2007b) studied the specific physiological role of TRPA1 channels. Using TRPA1-deficient mice, they discovered the key role of TRPA1 channels in chemical damage and pain sensing [Macpherson et al. (2007b), their Figs. 3 (http://www.jneurosci.org/ cgi/content/full/27/42/11412/F3), 4 (http:// www.jneurosci.org/cgi/content/full/27/42/ 11412/F4)]. The authors found that the formaldehyde-induced calcium influx or currents were completely abolished in cultured DRG neurons deficient in TRPA1 channels, suggesting that TRPA1 is the sole effector of the conductance induced by this nonselective reactive compound. Interestingly, the calcium influx and currents induced by several other compounds, including iodoacetamide (IA) and mustard oil, are also abolished in cells from TRPA1-deficient mice. Using a nociceptive behavior test on wild-type or TRPA1-deficient littermates, they also found that TRPA1-deficient mice displayed dramatically impaired nociceptive responses to injections of formaldehyde or IA. These data strongly suggest that TRPA1 channels play a central role in pain sensing, responding to a wide range of chemical compounds produced during tissue damage. It is plausible to propose that a single conductance may play a role as a "hub nociceptor" with wide specificity to chemicals in tissue damage and pain sensing (Fig. $1 B)$.

In summary, using a combination of several techniques, including calcium imaging, electrophysiological recording, and TRPA1-knock-out mice, this study reported exciting evidence about the gating property and physiological role of a TRP member, TRPA1. It is the first time that people identified an endogenous extracellular activator for TRP channels, although the detailed gating process is not yet clear. It should be noted, however, that some endogenous intracellular molecules can also potentiate some TRPs, such as DAG (to TRPC3/6/7), $\mathrm{Ca}^{2+}$ (to TRPM4/5), and cyclic ADP ribose (to TRPM2), but it is still elusive whether they directly activate TRPs (Venkatachalam and Montell, 2007). Macpherson et al. (2007b) also clearly proved that TRPA 1 plays a key role in pain sensing of chemical damage, using a TRPA1-knock-out mouse. They found that TRPA1 has broad specificity in vivo, suggesting that TRPA1 might underlie the central conductance activated during pain conditions. This study is exciting and should be a nice step toward clarifying the gating properties and specific physiologi-
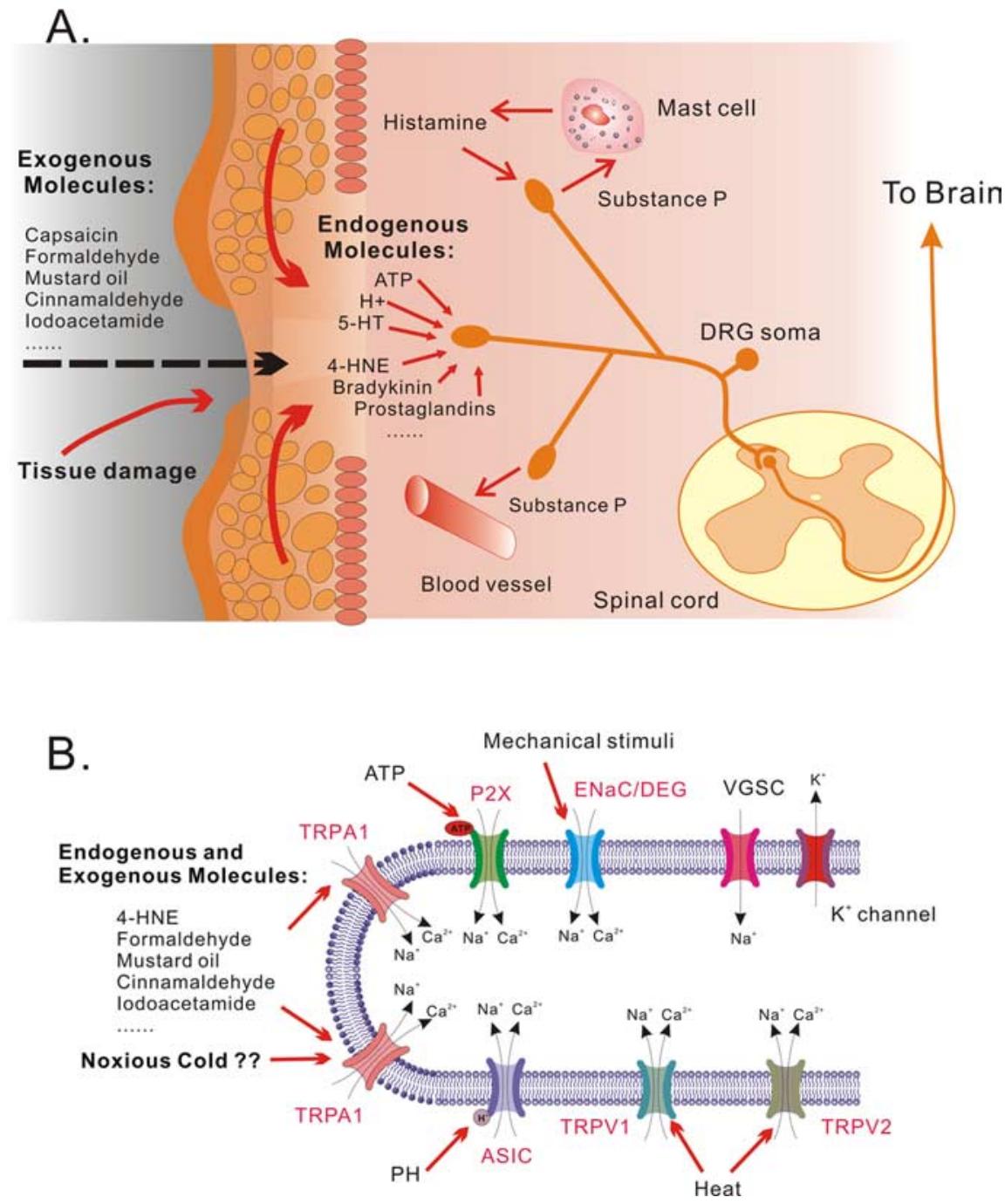

Figure 1. Schematic summary showing that TRPA1 plays a key role in chemical sensing in pain pathway. A, Overview of the nociceptive pain pathway. Some of the main components that are generated during tissue damage are shown, including peptides (bradykinin), lipids (prostaglandins), neurotransmitters (5-HT and ATP), 4-HNE, and $\mathrm{H}^{+}$. Each of these factors could depolarize and excite the terminals of the nociceptor by interacting with cell-surface receptors or ion channels expressed on the terminals. Activation of the nociceptor then transmits afferent messages to the spinal cord dorsal horn, and in turn to the brain. Nociceptors also release neurotransmitters like substance $P$ from the peripheral terminal, inducing vasodilation and plasma extravasation. Nociceptors can also activate many non-neuronal cells, including mast cells, which in turn release other chemicals into the extracellular space. This figure was adapted from Julius and Basbaum (2001). B, The key role of TRPA1 in chemical sensing. Nociceptors use a diversity of ion channels to detect physical and chemical stimuli: TRPV1 and TRPV2 detect noxious heat, ENaC/DEG channels detect mechanical stimuli, ASICs detect acid, and P2X detects ATP. TRPA1 might play a central role in detecting chemical signals, and maybe noxious cold.

cal role of other members of the TRP family.

Several questions not fully answered in this paper require future work. First, the detailed mechanisms underlying the gating processes are not clear. For example, we do not know whether the chemical compounds bind directly to the channels as ligands. Are there other receptors for these chemicals in the membrane that are the actual sensors that in turn lead to TRPA1 activation? Recently in a Nature paper, the same group reported that noxious compounds (e.g., mustard oil and cinnamaldehyde) could activate TRPA1 channels through covalent modification of cysteines (Macpherson et al., 2007a). It is not clear, however, whether the action of formaldehyde and 4-HNE on TRPA1 is also dependent on covalent modification. Obviously, more studies are required before we could clarify the gating mechanisms of TRPA1 by the diverse components. Second, during tissue damage or inflammatory conditions, numerous endogenous compounds are produced. To confirm the "hub nociceptor" role of TRPA1, the effects of these other molecules on TRPA 1 channels must be investigated, and other endogenous molecules 
able to activate TRPA1 channels must be identified. A third question is whether TRPA1 channels are also involved in other types of pain, which could be tested using the TRPA1-deficient mice described in this paper. Finally, although the authors identified 4-HNE as the first endogenous activator of TRPA1 channels, the effects of 4-HNE on the single-channel properties of TRPA1 and in nociceptive behaviors should be investigated in the future.

\section{References}

Jordt SE, Bautista DM, Chuang HH, McKemy DD, Zygmunt PM, Hogestatt ED, Meng ID,
Julius D (2004) Mustard oils and cannabinoids excite sensory nerve fibres through the TRP channel ANKTM1. Nature 427:260-265. Julius D, Basbaum AI (2001) Molecular mechanisms of nociception. Nature 413:203-210.

Macpherson LJ, Dubin AE, Evans MJ, Marr F, Schultz PG, Cravatt BF, Patapoutian A (2007a) Noxious compounds activate TRPA1 ion channels through covalent modification of cysteines. Nature 445:541-545.

Macpherson LJ, Xiao B, Kwan KY, Petrus MJ, Dubin AE, Hwang S, Cravatt B, Corey DP, Patapoutian A (2007b) An ion channel essential for sensing chemical damage. J Neurosci 27:11412-11415.

Story GM, Peier AM, Reeve AJ, Eid SR, Mosbacher J, Hricik TR, Earley TJ, Hergarden AC,
Andersson DA, Hwang SW, McIntyre P, Jegla T, Bevan S, Patapoutian A (2003) ANKTM1, a TRP-like channel expressed in nociceptive neurons, is activated by cold temperatures. Cell 112:819-829.

Trevisani M, Siemens J, Materazzi S, Bautista DM, Nassini R, Campi B, Imamachi N, Andre E, Patacchini R, Cottrell GS, Gatti R, Basbaum AI, Bunnett NW, Julius D, Geppetti P (2007) 4-Hydroxynonenal, an endogenous aldehyde, causes pain and neurogenic inflammation through activation of the irritant receptor TRPA1. Proc Natl Acad Sci USA 104:13519-13524.

Venkatachalam K, Montell C (2007) TRP channels. Annu Rev Biochem 76:387-417. 